\title{
Therapy with Plasma Growth Factors: Controversies of Key at Cellular Therapy
}

\author{
Alcaraz JR* \\ Hematologist and Medical advisory of Bridging Biosciences, USA
}

*Corresponding author: J Alcaraz Rubio, Hematologist and Medical advisory of Bridging Biosciences, FRISCO, Texas, USA, Email: jesusalcaraz@telefonica.net
Opinion

Volume 3 Issue 2

Received Date: July 06, 2019

Published Date: July 12, 2019

DOI: $10.23880 /$ hij-16000140

\section{Opinion}

Multiple applications at plasma growth factors are given for example in the field of regenerative medicine for the treatment of incipient degenerative arthropathies, chondropathies, mencopathies and tendinopathies in Traumatology and Sports Medicine, dry eye and corneal ulcers in Ophthalmology, vascular ulcers and diabetic foot in General Surgery, the approach of pain refractory to conventional analgesic therapy in Anesthesia even as a coadjutant of Neuro-rehabilitation for cognitive improvement in patients with neurological diseases of ischemic-anoxic origin or dental bed reparation in Dentistry among others. However, most of these clinical indications have poor scientific evidence, based many of them on empirical personal experience of each author, or in the publication of isolated clinical cases or series of clinical cases at most.

The scarcity of adequately structured and designed clinical trials makes the efficacy of this type of biotechnology for many of the postulated clinical applications more than doubtful since we do not know in almost all cases the cellular and protein composition of the final product obtained. This can be explained by the existence of procurement procedures not accredited by the scientific literature and not been reproduced by other authors. Currently there are as many methods for obtaining growth factors as there are clinical uses, including several different procedures for the same clinical application, which makes them unreliable given the variable results obtained and that have not been tested by other scientific groups.
Nowadays the law of the different agencies of the drug, require previous serological tests in each patient to be able to manipulate the total blood obtained. In the same way, this manipulation must be carried out in a sterile environment, either under a laminar B-flow hood, if it is an open procedure or by meeting the manufacturer's requirements for the different filters used in case the blood handling procedure is closed. In any case, failure to comply with this primordial regulation entails the possibility of transmission of infectious-contagious pathogens parenterally, as in the case of conventional blood donation. Hence, the hematological scientific societies are establishing programs of control and traceability of this type of biotreatment. This could be solved by creating working groups within the different government agencies of the drug that are responsible for reviewing those procedures for obtaining growth factors more reproduced in the scientific literature, where both cellular composition and proteins obtained after manipulation is exhaustively known. From whole blood and use them in each of clinical application that wants to study to objectify the effects achieved and perform a correct monitoring of each patient.

The existence of procedures to obtain automated plasma growth factors could be a great scientific advance when it comes to having a useful, reliable and reproducible tool for any scientific work group that intends to study the utility for a specific disease or clinical application which would allow the design of highpowered scientific studies in the form of clinical trials and meta-analyzes. Today we have a biotechnology with a great clinical and potential application that is still to be 


\section{Haematology International Journal}

explored and exploited, from a serious, rigorous and scientific point of view, considered a medicine for human use by most of the government agencies of the drug, lacking a token technique since we do not have enough scientific evidence in the form of clinical trials and that may be the key to explain the mechanism of immunomodulation, regeneration and protectionapoptosis of the cell cycle in so-called somatic cell therapies with stem cells of different origin. 